Рубрика: неинвазивная аритмология

๑) О.Л. БОКЕРИЯ, А.А. АХОБЕКОВ, 2014

( АННАЛЫ АРИТМОЛОГИИ, 2014

УдК 616.12-008.313.2:615.22:616.12-089.168.1

DOI: 10.15275/annaritmol.2014.1.4 ФИБРИЛЛЯЦИИ ПРЕДСЕРДИЙ ПОСЛЕ КАРДИОХИРУРГИЧЕСКИХ ОПЕРАЦИЙ

Tип статьи: обзорная статья

О.Л. Бокерия, А.А. Ахобеков

ФГБУ «Научный центр сердечно-сосудистой хирургии им. А.Н. Бакулева»

(директор - академик РАН и РАМН Л.А. Бокерия) РАМН; Рублевское шоссе, 135, Москва, 121552,

Российская Федерация

Бокерия Ольга Леонидовна, доктор мед. наук, профессор, гл. научн. сотр., зам. заведующего отделением; Ахобеков Альберт Амалиевич, аспирант, e-mail: alber-t7@mail.ru

Фибрилляция предсердий (ФП) - это наиболее частое нарушение ритма сердиа, возникающее после кардиохирургических операций. Частота развития ФП в раннем послеоперационном периоде при 
трансплантации сердца составляет около 11\%, при аортокоронарном шунтировании - 25-30\%, при коррекции клапанной патологии - 33-49\%, при сочетанных операциях - до 60\%. Около 95\% случаев развития ФП приходится на первые 6 сут после операции. Возникновение ФП ассоциируется с высоким риском развития инсульта, инфаркта миокарда, с послеоперационной летальностью, а также более длительным сроком госпитализации и, соответственно, большими экономическими затратами.

К послеоперационным факторам риска развития ФП относятся: воспаление, обусловленное посткардиотомным синдромом и искусственным кровообращением (ИК), электролитный дисбаланс, дисбаланс жидкости, длительная искусственная вентиляция легких, позднее отхождение от кардиотонической поддержки. Несмотря на многочисленные исследования, патофизиология возникновения ФП в раннем послеоперационном периоде после кардиохирургических операций остается до кониа не изученной.

В настоящее время воспаление как один из основных факторов риска развития ФП вызывает большой интерес у исследователей. Сходство по времени между пиком возникновения ФП после операций на сердие и активации системы комплемента с выпуском провоспалительных иитокинов (3-6-е сутки после операции) предполагает наличие воспалительного компонента в механизме запуска послеоперационной ФП. В связи с данной гипотезой ряд ученых уделяют внимание исследованию эффективности препаратов с противовоспалительными свойствами в профилактике послеоперационной ФП, касающейся операций на открытом сердие. В частности, последние несколько лет активно изучают эффективность статинотерапии. Ее положительный эффект связан $c$ плейотропными (противовоспалительными, антиоксидантными, мембраностабилизирующими) свойствами статинов.

Исследование ARMYDA-3 (Atorvastatin for Reduction MYocardial Dysrhythmia After cardiac surgery) первое исследование, в котором было показано, что предоперационное назначение аторвастатина в дозе 40 мг снижало частоту возникновения ФП после кардиохирургических операций.

Для получения более мощной доказательной базы в отношении эффективности статинотерапии в профилактике послеоперационной ФП необходимо проведение более крупных рандомизированных клинических исследований для каждого вида кардиохирургических операций.

Ключевые слова: фибрилляция предсердий; кардиохирургия; статины.

\section{THE EFFICIENCY OF STATINS IN THE PREVENTION OF ATRIAL FIBRILLATION AFTER CARDIAC OPERATIONS}

\section{O.L. Bockeria, A.A. Akhobekov}

A.N. Bakoulev Scientific Center for Cardiovascular Surgery of Russian Academy of Medical Sciences; Rublevskoe shosse, 135, Moscow, 121552, Russian Federation

Bockeria Ol'ga Leonidovna, MD, DM, Professor, Chief Research Associate, Deputy Chief of Department; Akhobekov Al'bert Amalievich, Postgraduate, e-mail: alber-t7@mail.ru

Atrial fibrillation $(A F)$ is the most frequent arrhythmia, occurring after heart surgery. The incidence of AF in early postoperative period after heart transplantation is about 11\%, after coronary artery bypass grafting $25-30 \%$, after correction of valvular pathology - 33-49\%, after combined operations - up to 60\%. About 95\% of cases the initiation of AF falls on the first 6 days after the operation. The emergence of AF is associated with a higher risk of stroke, myocardial infarction, postoperative mortality, longer hospital stays and, therefore, larger economic costs.

Postoperative risk factors for AF include inflammation caused by postcardiotomy syndrome and extracorporeal circulation, electrolyte imbalance, an imbalance of fluids, long-term artificial pulmonary ventilation, later discharge from cardiotonic support. Despite numerous studies, pathophysiology of occurrence of AF in the early postoperative period after cardiac operations remains to be elucidated.

Nowadays, inflammation, as one of the main risk factors for AF is a reason of a great interest of many researchers. The similarity of time between the peak of occurrence of AF after cardiac operations and activation of the complement system with the release of pro-inflammatory cytokines ( 3 to 6 days after surgery) implies the presence of inflammatory component in the mechanism of launching of postoperative AF. Because of this hypothesis, the attention of a number of researchers was focused on the studying the effectiveness of drugs with anti-inflammatory properties in prophylaxis of postoperative AF concerning operations on the open heart. In particular, during the last few years the effectiveness of statin therapy is actively studied. Its positive effect is associated with pleiotropic (anti-inflammatory, antioxidant and membrane-stabilizing properties of statins. Its positive effects are associated with pleiotropic (anti-inflammatory, antioxidant and membrane-stabilizing properties of statins.

The study ARMYDA-3 (Atorvastatin for Reduction MYocardial Dysrhythmia After cardiac surgery) is the first study in which it was demonstrated that preoperative administration of atorvastatin dose of $40 \mathrm{mg}$ reduced the incidence of AF after cardiac operations.

For more powerful evidence base on the effectiveness of statin therapy in prophylaxis of postoperative AF it is necessary to conduct a larger randomized clinical studies for each type of cardiac operations.

Key words: atrial fibrillation; cardiac surgery; statins. 


\section{Введение}

$\Phi$ ибрилляция предсердий (ФП) - это наиболее частое нарушение ритма сердца, развивающееся у пациентов, перенесших операцию на открытом сердце. Так, частота развития ФП в раннем послеоперационном периоде после операции трансплантации сердца составляет около $11 \%$, после аортокоронарного шунтирования - 25-30\%, после хирургической коррекции клапанной патологии - 33-49\%, при сочетанных операциях - до 60\%. Около $95 \%$ случаев развития ФП происходит в течение первых 6 сут после операции $[1,2]$. При впервые возникшей ФП у $80 \%$ больных удается восстановить синусовый ритм в течение первых суток, а у $98 \%-$ в течение ближайших 6 нед [3].

Возникновение ФП ассоциируется с высоким риском развития инсульта, инфаркта миокарда и послеоперационной летальности, а также с более длительным сроком госпитализации и, соответственно, значимо большими экономическими затратами. Так, в исследовании, включавшем 16169 пациентов, перенесших аортокоронарное шунтирование (АКШ), было показано, что возникновение ФП в раннем послеоперационном периоде являлось независимым предиктором послеоперационной летальности (относительный риск $(\mathrm{OP})-1,2)$ в течение шестилетнего периода наблюдения [1]. Риск развития инсульта был в 3 раза выше у пациентов с впервые возникшей ФП, чем у пациентов без нее. Длительность госпитализации у данной категории пациентов, по различным данным, увеличивалась на 3-4 дня, что в США вело к дополнительным экономическим затратам в размере 2 млрд долларов в год [1].

Таким образом, профилактика ФП у пациентов, переносящих операции на открытом сердце, актуальна и с медицинской, и с экономической точек зрения.

\section{Основные факторы риска развития послеоперационной фибрилляции предсердий}

Предоперационные факторы. Наиболее изученным фактором риска является возраст пациентов. J.P. Mathew и соавт. в своем исследовании показали, что пациенты старше 70 лет имеют самый высокий риск развития ФП. С возрастом происходят дегенеративные и воспалительные изменения в предсердиях, меняя их электрофизиологию. Изменение электрофизиологических свойств миокарда предсердий проявляется в том, что укорачивается эффективный рефрактерный период, развивается дисперсия проводимости и рефрактерности, создаются условия для аномального автоматизма, что в конечном итоге приводит к возникновению послеоперационной ФП [4]. Также к основным факторам риска относятся: ФП в анамнезе, врожденные или приобретенные пороки клапанов сердца, увеличение размеров левого предсердия, окклюзия или гемодинамически значимый стеноз правой коронарной артерии, фракция выброса левого желудочка менее $40 \%$, хроническая обструктивная болезнь легких, хроническая почечная недостаточность, сахарный диабет, метаболический синдром [5-7].

Интраоперационные факторы. Из интраоперационных факторов риска следует отметить выполнение операций в условиях искусственного кровообращения (ИК), кардиоплегию, длительное пережатие аорты, бикавальную канюляцию, сочетанные операции на сердце [8].

Послеоперационные факторы. К послеоперационным факторам риска относятся: воспаление, обусловленное посткардиотомным синдромом и ИК, электролитный дисбаланс, дисбаланс жидкости, длительная искусственная вентиляция легких, позднее отхождение от кардиотонической поддержки [9].

Несмотря на многочисленные исследования, патофизиология развития ФП после кардиохирургических операций до конца не изучена. Сложность выделения основного механизма связана с многофакторностью ее формирования (рис. 1).

В настоящее время воспаление как один из основных факторов риска развития ФП вызывает большой интерес у исследователей. Сходство по времени между пиком возникновения ФП после операций на сердце и активации системы комплемента с выпуском провоспалительных цитокинов (3-6-е сутки после операции) предполагает наличие воспалительного компонента в механизме запуска послеоперационной ФП [10]. На активацию системы комплемента и воспаления указывает увеличение содержания в крови таких маркеров, как С-реактивный белок, интерлейкин-2, интерлейкин-6, лейкоциты [11-14].

Активация системы комплемента при операциях с ИК происходит в два этапа. Первый этап запускается непосредственно во время проведения ИК и является результатом взаимодействия 
Предоперационные факторы

Пожилой возраст

Артериальная гипертензия

Сахарный диабет

Ожирение

Метаболический синдром

Расширение левого предсердия

Диастолическая дисфункция

Гипертрофия левого желудочка
Интраоперационные факторы

Хирургические повреждение

предсердий

Ишемия предсердий

Бикавальная канюляция

Кардиоплегия
Послеоперационные факторы

Воспаление

Оксидантный стресс

Дисбаланс жидкости

Дисбаланс автономной нервной

системы

Электролитный дисбаланс

(гипомагниемия, гипокалиемия)
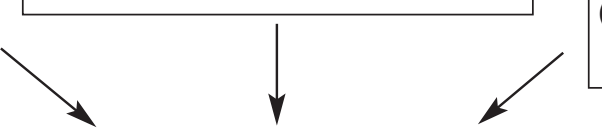

Структурный субстрат для возникновения фибрилляции предсердий

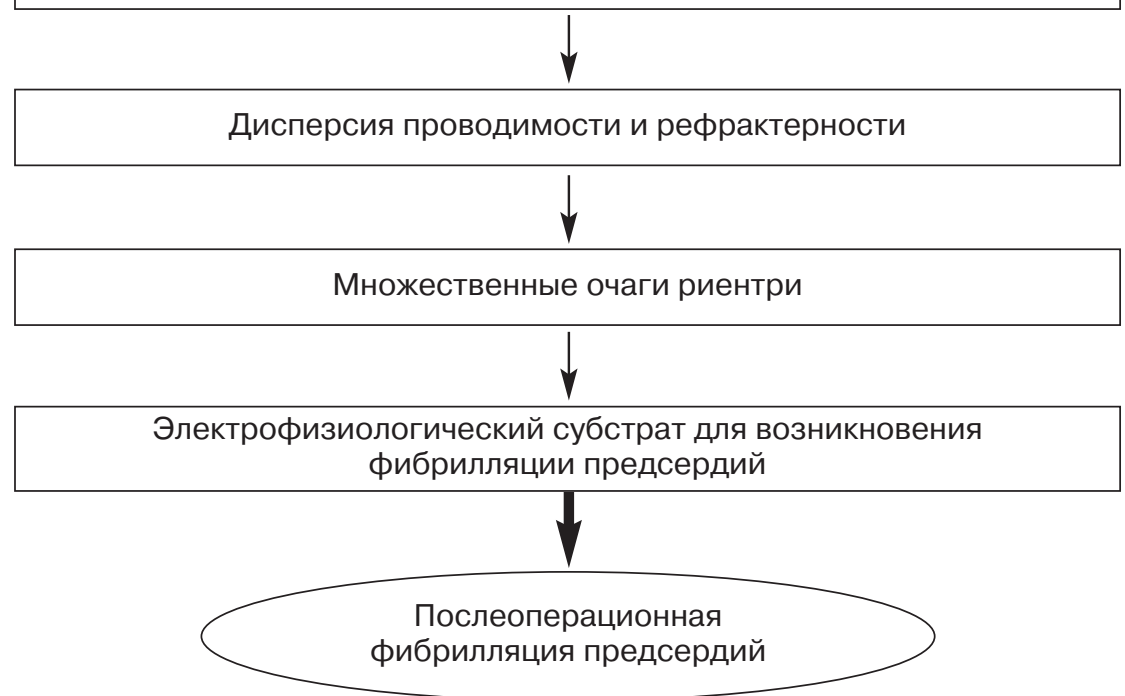

Рис. 1. Пре-, интра- и послеоперационные факторы риска развития послеоперационной фибрилляции предсердий

крови с поверхностью экстракорпорального контура или опосредованно - через «альтернативный путь» с участием фактора некроза опухоли $\alpha$. Второй этап развивается по «классическому пути» и связан с введением протамина после ИК. Помимо системной воспалительной реакции, вызванной ИК, местное воспаление, возникающее вследствие хирургического воздействия, тоже играет важную роль в развитии послеоперационной ФП. Известно, что степень предсердного воспаления повышается с увеличением инвазивности хирургического вмешательства, но даже после перикардиотомии предсердия вовлекаются в воспаление.

Сравнение частоты развития послеоперационной ФП после кардиохирургических операций в условиях ИК и без него позволило выявить роль системного и местного воспаления. Многие исследования не смогли показать статистически значимой разницы между двумя группами [15]. Данный факт дает возможность предполагать, что обусловленное посткардиотомным синдромом местное воспаление, в отличие от системного, является более существенным фак- тором при возникновении послеоперационной ФП. Однако следует отметить, что во всех исследованиях показана тенденция к снижению частоты послеоперационной ФП при кардиохирургических операциях, когда ИК не применялось. В настоящее время ряд исследователей уделяют большое внимание изучению эффективности препаратов с противовоспалительными свойствами в профилактике ФП после операций на открытом сердце. В частности, последние несколько лет активно изучается статинотерапия. Ее положительный эффект связан с плейотропными (противовоспалительными, антиоксидантными, мембраностабилизирующими) свойствами статинов [16, 17].

\section{Статины в профилактике послеоперационной фибрилляции предсердий}

Исследование ARMYDA-3 (Atorvastatin for Reduction MYocardial Dysrhytmia After cardiac surgery) - первое исследование, в котором было показано, что предоперационное назначение аторвастатина в дозе 40 мг снижало частоту воз- 
никновения ФП после кардиохирургических операций. Исследование ARMYDA-3 являлось рандомизированным, проспективным, двойным слепым, плацебоконтролируемым клиническим испытанием, проводившимся на базе биомедицинского университета в Италии (г. Рим). В исследовании участвовали 200 пациентов после операций на остановленном сердце. Из них 99 получали плацебо, и 101 - аторвастатин в дозе 40 мг/сут. Лечение начиналось за 7 дней до плановой операции и продолжалось после операции.

В качестве первичной конечной точки исследования была принята частота возникновения ФП после операции в госпитальном периоде. Вторичные конечные точки исследования: 1) сроки пребывания в стационаре после операций в двух сравниваемых группах; 2) частота серьезных сердечных и цереброваскулярных осложнений (смерть, инсульт, инфаркт миокарда или необходимость коронарной реваскуляризации) с момента операции и в течение 30 дней после операции в двух группах; 3) взаимосвязь послеоперационного пика уровня С-реактивного белка (СРБ) и частоты ФП; 4) определение основных предикторов ФП.

Фибрилляция предсердий после операций возникла у 35 (35\%) из 101 пациента в группе аторвастатина против 56 (57\%) из 99 пациентов группы плацебо $(p=0,003)$. Средние сроки пребывания в стационаре после операций оказались короче в группе принимавших аторвастатин по сравнению с группой плацебо $(6,3 \pm 1,2$ против 6,9 $\pm 1,4$ дня, $p=0,001)$. По данным многофакторного анализа, лечение аторвастатином было связано со снижением риска развития ФП после операций на сердце на $61 \%$ (отношение шансов (ОШ) 0,39, 95\% доверительный интервал (ДИ) 0,18-0,85, $p=0,017)$, а послеоперационные пиковые уровни СРБ, превышавшие средние значения (166 мг/л), сопровождались высоким риском развития ФП (ОШ 2,0, $95 \%$ ДИ $1,2-7,0, p=0,01)$. Возраст старше 65 лет, системная артериальная гипертензия и атеросклероз аорты также являлись предикторами возрастания риска, тогда как применение бета-адреноблокаторов давало профилактический эффект (ОШ 0,19, 95\% ДИ 0,08-0,44, $p=0,0001$ ) [18].

Группа авторов из кардиологической клиники университета г. Аликанте в 2006 г. опубликовала интересные результаты работы по данной теме. В исследование были включены 234 пациента после АКШ в возрасте 65土9 лет, у которых оценивали влияние статинов на уровень матриксной металлопротеиназы-1 (ММР-1) и тканевого ингибитора матриксной металлопротеиназы-1 (ТІМР-1) в плазме крови и на частоту возникновения послеоперационной ФП. Металлопротеиназа-1 входит в семейство цинкзависимых эндопептидаз, обладающих внеклеточной активностью, и имеет способность разрушать все компоненты внеклеточного матрикса. Лейкоциты, большей частью макрофаги, являются основным источником ММР-1, которая способствует экстравазации и проникновению белых кровяных клеток в ткани. Данное явление, в свою очередь, лежит в основе развития воспалительного процесса. ТІМР - это белки, поддерживающие баланс между деструкцией и формированием матрикса путем специфического ингибирования ММР.

Из 234 пациентов у 66 (28,2\%) развилась послеоперационная ФП. При многофакторном анализе данных выяснилось, что статинотерапия была связана с уменьшением частоты развития ФП (ОШ 0,52, 95\% ДИ 0,28-0,96, $p=0,038)$. Индекс TIMP-1/MМР-1 через 24 ч после операции был выше у пациентов без ФП $(p=0,043)$. Статинотерапия сопровождалась повышением уровня ТІМР-1 и индекса TIMP-1/MМР-1 ( $p=0,027$ и 0,036 соответственно) [19]. В данной работе были подробно описаны роль воспаления в развитии послеоперационной ФП и механизм противовоспалительного эффекта статинотерапии.

G. Mariscalco и соавт. также продемонстрировали положительное влияние статинов на частоту развития послеоперационной ФП у пациентов после АКШ. Так, в результате анализа данных 405 пациентов, перенесших АКШ, было показано, что предоперационная статинотерапия снижает риск развития ФП на $43 \%$ и одинаково эффективна в различных возрастных группах.

Группа авторов из Хартфордского госпиталя в 2008 г. впервые показала, что добавление статинов к уже проводимой антиаритмической терапии у пациентов с высоким риском развития послеоперационной ФП помогает эффективно снижать число послеоперационных аритмических осложнений. Данными исследователями проанализированы 555 пациентов после различных операций на открытом сердце, 331 из которых получал статины, а 224 - нет. Средний возраст пациентов составил $67,8 \pm 8,6$ года, $77,1 \%$ из них были мужчины, 14,6\% пациентам была 
проведена коррекция клапанной патологии, 6,1\% - имели ФП в анамнезе, 84\% - принимали бета-адреноблокаторы после операции, 44,1\% кордарон. В результате у $174(31,4 \%)$ пациентов возникла послеоперационная ФП. В соответствии с мультивариантным анализом данных статинотерапия ассоциировалась с уменьшением частоты послеоперационной ФП (ОШ 0,60; 95\% ДИ 0,37-0,99). У пациентов, получавших бетаадреноблокаторы и кордарон, статинотерапия дополнительно снижала частоту развития ФП на $40 \%$ [20].

F. Rader и соавт. в 2011 г. представили работу, в которой показали, что раннее послеоперационное назначение статинов способствует профилактике ФП. В исследование было включено 200 пациентов после операций аортокоронарного шунтирования и коррекции клапанной патологии. Пациенты, получавшие оптимальную медикаментозную терапию, согласно принятым рекомендациям, были разделены на две группы: пациенты со статинотерапией и без нее. В раннем послеоперационном периоде пароксизмы ФП наблюдались у 36 (18\%) пациентов. В первой группе это были всего 4 (7,7\%) пациента, а во второй -32 (28\%), $p=0,043$ [21] .

В то же время было опубликовано несколько работ, в которых показано, что после статинотерапии не произошло статистически значимого снижения риска развития послеоперационной ФП. Одна из таких работ, которая включала 4044 пациента, была представлена в 2007 г. S.V. Salim и соавт. Стоит отметить, что пациенты в данном исследовании не получали статины в послеоперационном периоде.

В связи с противоречивыми результатами исследований очень важно проведение метаанализов и систематических обзоров для определения реального места статинов в профилактике послеоперационной ФП.

Первый такой метаанализ был опубликован в 2009 г. группой авторов из Университетской больницы города Кельна. В метаанализ вошли 13 исследований (из них 3 рандомизированных клинических исследования), которые проводились в 2004-2008 гг. и включали 17643 пациентов, перенесших операции на открытом сердце. Из них 10304 (58\%) пациента получали статины, а 7339 (42\%) - нет. В 8 исследованиях проводился анализ результатов наблюдения за пациентами, перенесшими операцию аортокоронарного шунтирования, которые составили 90,7\% всех включенных в метаанализ пациентов.
В 5 оставшихся исследованиях были изучены результаты наблюдения за пациентами, перенесшими хирургическую коррекцию клапанной патологии и комбинированные хирургические процедуры. Эти больные составили 6,5 и 2,8\% соответственно.

Во всех исследованиях использовались различные дозы статинов и схемы их назначения. Заболеваемость ФП для всей популяции пациентов составила 24,6\% (4335 из 17643 пациента) и была значительно ниже у пациентов, получающих предоперационную статинотерапию по сравнению с контрольной группой (22,3\% против $27,8, p<0,0001)$. Применение статинов привело к снижению абсолютного $(5,5 \%)$ и относительного (19,9\%) рисков развития послеоперационной ФП. Анализ подгрупп с изучением частоты развития впервые возникшей ФП в 9 исследованиях показал высокую послеоперационную заболеваемость - 29,9\% (2349 из 7855 пациентов). Предоперационное применение статинов привело к значительному снижению риска развития впервые возникшей ФП по сравнению с плацебо (28,2\% против 32,$2 ; p=0,0001)$ со снижением абсолютного риска на $4 \%$, относительного - на 12,4\%. Результаты метаанализа свидетельствовали об эффективности статинотерапии в первичной и вторичной профилактике послеоперационной ФП [22].

В другом метаанализе, опубликованном в 2010 г., оценивалась зависимость эффективности статинотерапии в профилактике послеоперационной ФП от применяемых доз и продолжительности ее предоперационного проведения. В метаанализе проводился анализ результатов 8 исследований $(n=774) ; 389$ пациентов были включены в группу статинотерапии, 385 пациентов - в контрольную группу. В исследованиях использовались следующие статины и их дозировки: аторвастатин (20 и 40 мг), флувастатин (80 мг), правастатин (40 мг), розувастатин (20 мг) и симвастатин (20 мг). Продолжительность предоперационной статинотерапии колебалась от 2 до 28 дней. Регрессионный анализ показал связь между продолжительностью предоперационной статинотерапии и риском развития послеоперационной ФП в виде снижения абсолютного риска на $3 \%$ в день $(p=0,008)$. Никакой связи между дозой используемых статинов и снижением риска развития послеоперационной ФП выявлено не было $(p=0,47)$ [23].

Стоит отметить, что результаты данного метаанализа противоречат результатам других 
крупных рандомизированных кинических исследований AFFIST I, II, III (Atrial Fibrillation Suppression Trials), цель которых заключалась в оценке влияния препаратов с антиаритмическим эффектом на риск развития послеоперационной ФП. В этих исследованиях было показано, что использование высоких доз статинов $(\geqslant 40$ мг аторвастатина - эквивалентная доза) сопряжено с большей эффективностью. Авторы данного метаанализа указали на то, что полученные результаты в отношении эффективности различных доз статинов могут быть связаны с недостаточной масштабностью исследования [24-26].

Систематический обзор с анализом 63 клинических исследований, включавшим 27363 больных, проведенный в период с 1980 по 2012 г., был опубликован в 2014 г. В работе определяли прогностическую ценность различных сывороточных маркеров воспаления и эффективность статинотерапии в профилактике послеоперационной ФП, частота развития которой составила 25,5\%. Повышенные уровни СРБ, интерлейкина 2, 6, 8 и лейкоцитов были связаны с высоким риском развития ФП, но наиболее убедительная связь была обнаружена с высоким уровнем лейкоцитов. Из всех лекарственных средств с противовоспалительными свойствами статины оказались самыми эффективными в профилактике послеоперационной ФП. После статинов по эффективности следовали: антиоксиданты (витамин C, N-ацетилцистеин, омега-3 полиненасыщенные жирные кислоты), стероиды (дексаметазон) и колхицин. Нестероидные противо- воспалительные препараты не показали статистически значимой эффективности в профилактике послеоперационной ФП. Несмотря на масштабность данного систематического обзора, авторы указывают, что большим недостатком является отсутствие в их работе метаанализа. Включенные в обзор исследования оказались гетерогенными в клинических и статистических аспектах, что помешало в проведении метаанализа. Исследования охватывали большой период (более 30 лет), в течение которого многие технические и тактические аспекты в кардиохирургии поменялись, что также определяет значительную неоднородность исследований [27].

Самый масштабный на сегодняшний день метаанализ клинических исследований, посвященный предоперационной статинотерапии, представлен 54 исследованиями, которые проводились в период между 1999 и 2011 гг., включал 91491 пациента (рис. 2). Двенадцать из 54 исследований являлись рандомизированными клиническими исследованиями с общим числом больных 1041 (1,1\%). В группу статинотерапии были включены 46614 (50,9\%) пациентов, в контрольную группу - 44877 (49,1\%). Большинство исследований были сосредоточены на изолированных АКШ ( $n=44 ; 62109$ больных; 67,9\%), в то время как операции на клапанах или комбинированные операции были выполнены в 20,6 или $11,5 \%$ случаев соответственно.

Использовались различные статины, их дозировки и продолжительность предоперацион-

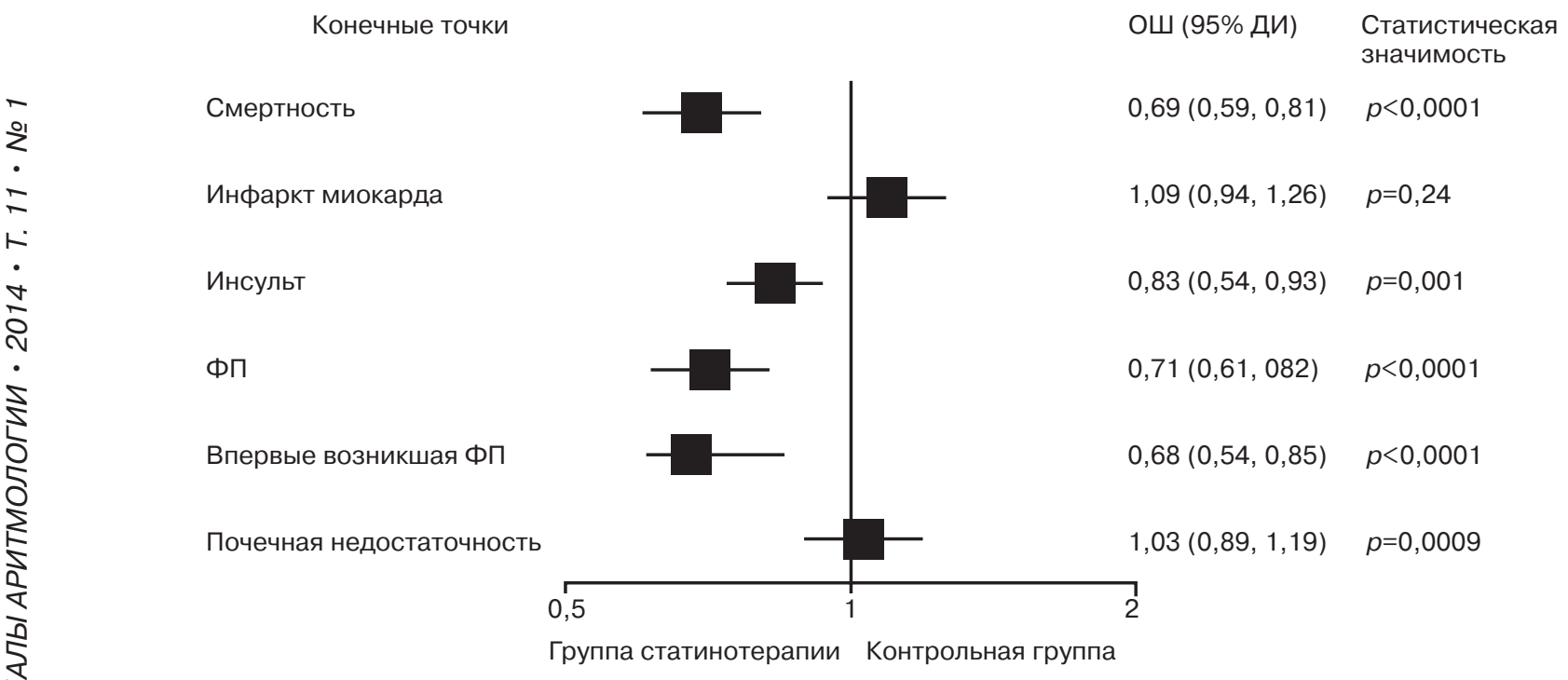

Рис. 2. Влияние статинотерапии на основные конечные точки после кардиохирургических операций 
ной терапии. Конечными точками для изучения в этом метаанализе были: смертность, ишемический инсульт, ФП, острый инфаркт миокарда и почечная недостаточность. Предоперационная статинотерапия привела к снижению абсолютного риска ранней смертности от всех причин на $0,9 \%$ (2,6\% против 3,5$)$ и относительного риска на 24,4\% (ОР 0,69, 95\% ДИ 0,59-0,81; $p<0,0001)$. Также статинотерапия была сопряжена со снижением абсолютного риска развития ишемического инсульта на $0,77 \%$ и относительного риска на 26,3\% (OP 0,83; $95 \%$ ДИ 0,74-0,93; $p=0,001)$. Статинотерапия привела к снижению относительного риска развития послеоперационной ФП на $31 \%$. Не удалось выявить статистически значимых различий между двумя группами в отношении инфаркта миокарда и почечной недостаточности. Таким образом, результаты данного метаанализа свидетельствуют о положительном влиянии предоперационной статинотерапии в отношении смертности, ишемического инсульта и ФП [28, 29].

Группой авторов из Шанхайского университета в 2014 г. опубликован самый новый метаанализ исследований по эффективности статинотерапии в профилактике ФП после операций АКШ [30]. В метаанализ были включены 12 исследований, из них 4 рандомизированных и 8 обсервационных, с общим количеством пациентов 8458 . Из них 5120 (60,53\%) получали предоперационную статинотерапию, а 3338 $(39,47 \%)$ - нет. Возраст пациентов, распространенность мужского пола, дислипидемия, застойная сердечная недостаточность, перенесенный инфаркт миокарда, артериальная гипертензия, сахарный диабет, фракция выброса левого желудочка в двух исследуемых группах были сопоставимы. В трех исследованиях с плацебоконтролируемыми группами в группе статинотерапии частота развития ФП составила $16,8 \%$ по сравнению с $35,9 \%$ в группе плацебо $(p<0,00001)$. В 9 исследованиях с обычными контрольными группами - 16,5 и 20,3\% соответственно $(p=0,005)$.

Для оценки потенциального влияния условий выполнения АКШ были отдельно проанализированы исследования, в которых операции проводились: 1) только на работающем сердце; 2) на работающем и на остановленном сердце; 3) только на остановленном сердце. В 3 исследованиях, которые изучали АКШ на работающем сердце, частота послеоперационной ФП составила $13,7 \%$ в группе статинотерапии по сравне- нию с 26,8\% в контрольной группе $(p<0,00001)$. В 8 исследованиях, в которых АКШ выполнялось как на работающем, так и на остановленном сердце, послеоперационная ФП возникала реже в группе статинотерапии, чем в контрольной группе (16,8\% по сравнению с 20,3\%, $p<0,0001)$. И в 1 исследовании были проанализированы данные пациентов, перенесших АКШ на остановленном сердце. Было установлено, что частота развития ФП составила в группе статинотерапии 18,4 , в контрольной группе $41,2 \%(p=0,01)$.

С целью определения оптимальных сроков предоперационной статинотерапии сравнивали результаты пациентов, которым статины назначали за 7 дней до операции и за 1 день. Полученные данные свидетельствуют о большей эффективности статинотерапии в профилактике ФП при ее более раннем назначении (16,2\% по сравнению с 18,9\%, $p=0,01)$.

Также была выявлена польза статинотерапии в отношении сокращения сроков стационарного лечения пациентов после АКШ $(p=0,01)$.

\section{Заключение}

На сегодняшний день, несмотря на оптимальную медикаментозную терапию, риск развития ФП после операций на открытом сердце колеблется от 10 до $60 \%$ в зависимости от вида операции. Вероятно, это связано с недостаточными мерами профилактики из-за ограниченных знаний о причинах развития послеоперационной ФП. Результаты представленных в данной статье исследований свидетельствуют о несомненной роли воспаления в развитии послеоперационной ФП. Доказательством этому служит связь высокого уровня различных маркеров воспаления с возникновением послеоперационной ФП. Во многих клинических исследованиях продемонстрирована эффективность статинов в профилактике послеоперационной ФП через противовоспалительные и антиоксидантные механизмы.

Благодаря полученным результатам, в соответствии с современными международными рекомендациями статины были включены в список обязательных препаратов, которые должны принимать пациенты до и после операции АКШ с целью снижения послеоперационных осложнений. В профилактике ФП высокие дозы статинов более эффективны, чем низкие. Назначение статинов за 5-7 дней до операции эффективнее, чем их назначение после операции или 
за день до операции. Для получения более мощной доказательной базы в отношении эффективности статинотерапии в профилактике послеоперационной ФП необходимо проведение более крупных рандомизированных клинических исследований для каждого вида кардиохирургических операций.

\section{Конфликт интересов}

\section{Конфликт интересов не заявляется.}

\section{Библиографический список}

1. Echahidi N., Pibarot P., O'Hara G., Mathieu P. Mechanisms, prevention and treatment of atrial fibrillation after cardiac surgery. J. Am. Coll. Cardiol. 2008; 51: 793-801. DOI: 10.1016/j.jacc.2007.10.043.

2. Кандинский М.Л., Козлов Б.Н., Антонченко И.В. и др. Возникновение суправентрикулярных нарушений ритма сердца у больных в раннем послеоперационном периоде при аортокоронарном шунтировании. Progr. Biomed. Res. 2000; 5 (2), Suppl. A: 1-6.

3. El-Chami M.F., Kilgo P., Thourani V. et al. New onset atrial fibrillation predicts long term mortality after coronary artery bypass graft. J. Am. Coll. Cardiol. 2010; 55: 1370-6. DOI: $10.1016 /$ j.jacc.2009.10.058.

4. Mathew J.P., Fontes M.L., Tudor I.C. et al. A multicenter risk index for atrial fibrillation after cardiac surgery. JAMA. 2004; 291 (14): 1720-9. DOI: 10.1001/jama.291.14.1720.

5. Шварц В.А., Киселев А.Р., Петунина И.Э. Электролитные нарушения и их коррекция у кардиохирургических больных. Русский медицинский журнал. 2013; 21 (12): 617-20.

6. Бокерия Л.А., Голухова Е.З., Алшибая М.Д. и др. Прогнозирование развития фибрилляции предсердий после операций аортокоронарного шунтирования. Анналы аритмологии. 2004; 1 (1): 78-86.

7. Auer J., Weber T., Berent R. et al. Risk factors of postoperative atrial fibrillation after cardiac surgery. J. Card. Surg. 2005; 20 (5): 425-31. DOI: $10.1111 / \mathrm{j} .1540-8191.2005 .2004123 . x$

8. Aranki S.F., Shaw D.P., Adams D.H. et al. Predictors of atrial fibrillation after coronary artery surgery current trends and impact on hospital resources. Circulation. 1996; 94: 390-7. DOI: 10.1161/01.CIR.94.3.390.

9. White C.M., Caron M.F., Kalus J.S. et al. Intravenous plus oral amiodarone, atrial septal pacing, or both strategies to prevent post-cardiothoracic surgery atrial fibrillation: The atrial fibrillation suppression trial II (AFIST II). Circulation. 2003; 108 (Suppl. 1): II200-6. DOI: 10.1161/01.cir.0000087445.59819.6f.

10. Anselmi A., Possati G., Gaudino M. Postoperative inflammatory reaction and atrial fibrillation: simple correlation or causation? Ann. Thorac. Surg. 2009; 88: 326-33. DOI: 10.1016/j.athoracsur.2009.01.031.

11. Gaudino M., Andreotti F., Zamparelli R. et al. The $-174 \mathrm{G} / \mathrm{C}$ interleukin-6 polymorphism influences postoperative interleukin-6 levels and postoperative atrial fibrillation. Is atrial fibrillation an inflammatory complication? Circulation. 2003; 108 (Suppl. 1): II195-9. DOI: 10.1161/ 01.cir.0000087441.48566.0d.

12. Hak Ł., Myśliwska J., Wickiewicz J. et al. Interleukin-2 as a predictor of early postoperative atrial fibrillation after cardiopulmonary bypass graft (CABG). J. Interferon Cytokine Res. 2009; 29 (6): 327-32. DOI: 10.1089/jir.2008.0082.2906.

13. Lamm G., Auer J., Weber T. et al. Postoperative white blood cell count predicts atrial fibrillation after cardiac surgery. J. Cardiothorac. Vasc. Anesth. 2006; 20 (1): 51-6. DOI: 10.1053/j.jvca.2005.03.026.

14. Chung M.K., Martin D.O., Sprecher D. et al. C-reactive protein elevation in patients with atrial arrhythmias: inflammatory mechanisms and persistence of atrial fibrillation. Circulation. 2001; 104 (24): 2886-91. DOI: 10.1161/hc4901.101760.

15. Scherer M., Sirat A., Dogan S. et al. Does totally endoscopic access for off-pump cardiac surgery influence the incidence of postoperative atrial fibrillation in coronary artery bypass grafting? A preliminary report. Cardiovasc. Engg. 2006; 6 (3): $118-21$.

16. Liakopoulos O.J., Choi Y.H., Kuhn E.W. et al. Statins for prevention of atrial fibrillation after cardiac surgery: a systematic literature review. J. Thorac. Cardiovasc. Surg. 2009; 138 (3): 678-86. DOI: 10.1016/j.jtcvs.2009.03.054.

17. Liakopoulos O.J., Kuhn E.W., Slottosch I. et al. Preoperative statin therapy for patients undergoing cardiac surgery. Cochrane Database Syst. Rev. 2012; 4: CD008493. DOI: 10.1002/14651858.CD008493.pub2.

18. Patti G., Chello M., Candura D. et al. Randomized trial of atorvastatin for reduction of postoperative atrial fibrillation in patients undergoing cardiac surgery: results of the ARMYDA-3 (Atorvastatin for Reduction of Myocardial Dysrhythmia After cardiac surgery) study. Circulation. 2006; 114 (14): 1455-61. DOI: 10.1161/CIRCULATIONAHA.106.621763.

19. Marín F., Pascual D.A., Roldán V. et al. Statins and postoperative risk of atrial fibrillation following coronary artery bypass grafting. Am. J. Cardiol. 2006; 97 (1): 55-60. DOI: 10.1016/j.amjcard.2005.07.124.

20. Lertsburapa K., White C.M., Kluger J. et al. Preoperative statins for the prevention of atrial fibrillation after cardiothoracic surgery. J. Thorac. Cardiovasc. Surg. 2008; 135 (2): 405-11. DOI: $10.1016 / \mathrm{j}$.jtcvs.2007.08.049.

21. Rader F., Gajulapalli R.D., Pasala T., Einstadter D. Effect of early statin therapy on risk of atrial fibrillation after coronary artery bypass grafting with or without concomitant valve surgery. Am. J. Cardiol. 2011; 108 (2): 220-2. DOI: 10.1016/ j.amjcard.2011.03.028.

22. Liakopoulos O.J. Statins for prevention of atrial fibrillation after cardiac surgery: a systematic literature review. J. Thorac. Cardiovasc. Surg. 2009; 138 (3): 678-86.e1. DOI: 10.1016/ j.jtcvs.2009.03.054.

23. Chen W.T. Effect of statins on atrial fibrillation after cardiac surgery: a duration- and dose-response meta-analysis. J. Thorac. Cardiovasc. Surg. 2010; 140 (2): 364-72. DOI: 10.1016/ j.jtcrs.2010.02.042.

24. Lertsburapa K., White C.M., Kluger J. et al. Preoperative statins for the prevention of atrial fibrillation after cardiothoracic surgery. J. Thorac. Cardiovasc. Surg. 2008; 135 (2): 405-11. DOI: $10.1016 /$ j.jtcvs.2007.08.049.

25. Kourliouros A., De Souza A., Roberts N. et al. Dose-related effect of statins on atrial fibrillation after cardiac surgery. Ann. Thorac. Surg. 2008; 85 (5): 1515-20. DOI: 10.1016/ j.athoracsur.2008.01.040.

26. Mithani S., Akbar M.S., Johnson D.J. et al. Dose dependent effect of statins on postoperative atrial fibrillation after cardiac surgery among patients treated with beta blockers. J. Cardiothorac. Surg. 2009; 4: 61. DOI: 10.1186/ 1749-8090-4-61.

27. Jacob K.A., Nathoe H.M., Dieleman J.M. et al. Inflammation in new-onset atrial fibrillation after cardiac surgery: a systematic review. Eur. J. Clin. Invest. 2014; 44 (4): 402-28. DOI: 10.1111/eci.12237.

28. Kuhn E.W., Liakopoulos O.J., Stange S. et al. Preoperative statin therapy in cardiac surgery: a meta-analysis of 90000 patients. Eur. J. Cardiothorac. Surg. 2014; 45 (1): 17-26. DOI: 10.1093/ejcts/ezt181.

29. Mariscalco G., Lorusso R., Klersy C. et al. Observational study on the beneficial effect of preoperative statins in reducing atrial fibrillation after coronary surgery. Ann. Thorac. Surg. 2007; 84 (4): 1158-64. DOI: 10.1016/j.athoracsur.2007.05.021.

30. Zheng H., Xue S., Hu Z.L. et al. The use of statins to prevent postoperative atrial fibrillation after coronary artery bypass grafting: a meta-analysis of 12 studies. J. Cardiovasc. Pharmacol. 2014.

\section{References}

1. Echahidi N., Pibarot P., O'Hara G., Mathieu P. Mechanisms, prevention and treatment of atrial fibrillation after cardiac surgery. J. Am. Coll. Cardiol. 2008; 51: 793-801. DOI: 10.1016/j.jacc.2007.10.043

2. Kandinsky M.L., Kozlov N.N., Antonchenko I.V. et al. The emergence of supraventricular arrhythmias in patients in the early postoperative period when CABG. Progr. Biomed. Res. 2000; 5 (2), Suppl. A: 1-6 (in Russian). 
3. El-Chami M.F., Kilgo P., Thourani V. et al. New onset atrial fibrillation predicts long term mortality after coronary artery bypass graft. J. Am. Coll. Cardiol. 2010; 55: 1370-6. DOI: 10.1016/j.jacc.2009.10.058

4. Mathew J.P., Fontes M.L., Tudor I.C. et al. A multicenter risk index for atrial fibrillation after cardiac surgery. JAMA. 2004; 291 (14): 1720-9. DOI: 10.1001/jama.291.14.1720.

5. Shvartz V.A., Kiselev A.R., Petunina I.E. Electrolyte disorders and their correction in cardiosurgical patients. Russkiy Meditsinskiy Zhurnal. 2013; 21 (12): 617-20 (in Russian).

6. Bockeria L.A., Golukhova E.Z., Alshibaya M.D. et al. Risk stratification of atrial fibrillation after coronary artery bypass surgery. Annaly Aritmologii. 2004; 1 (1): 78-86 (in Russian).

7. Auer J., Weber T., Berent R. et al. Risk factors of postoperative atrial fibrillation after cardiac surgery. J. Card. Surg. 2005; 20 (5): 425-31. DOI: 10.1111/j.1540-8191.2005.2004123.x.

8. Aranki S.F., Shaw D.P., Adams D.H. et al. Predictors of atrial fibrillation after coronary artery surgery current trends and impact on hospital resources. Circulation. 1996; 94: 390-7. DOI: 10.1161/01.CIR.94.3.390.

9. White C.M., Caron M.F., Kalus J.S. et al. Intravenous plus oral amiodarone, atrial septal pacing, or both strategies to prevent post-cardiothoracic surgery atrial fibrillation: The atrial fibrillation suppression trial II (AFIST II). Circulation. 2003; 108 (Suppl. 1): II200-6. DOI: 10.1161/01.cir.0000087445.59819.6f.

10. Anselmi A., Possati G., Gaudino M. Postoperative inflammatory reaction and atrial fibrillation: simple correlation or causation? Ann. Thorac. Surg. 2009; 88: 326-33. DOI: 10.1016/ j.athoracsur.2009.01.031.

11. Gaudino M., Andreotti F., Zamparelli R. et al. The $-174 \mathrm{G} / \mathrm{C}$ interleukin-6 polymorphism influences postoperative interleukin-6 levels and postoperative atrial fibrillation. Is atrial fibrillation an inflammatory complication? Circulation. 2003; 108 (Suppl. 1): II195-9. DOI: 10.1161/ 01.cir.0000087441.48566.0d.

12. Hak Ł., Myśliwska J., Wickiewicz J. et al. Interleukin-2 as a predictor of early postoperative atrial fibrillation after cardiopulmonary bypass graft (CABG). J. Interferon Cytokine Res. 2009; 29 (6): 327-32. DOI: 10.1089/jir.2008.0082.2906.

13. Lamm G., Auer J., Weber T. et al. Postoperative white blood cell count predicts atrial fibrillation after cardiac surgery. J. Cardiothorac. Vasc. Anesth. 2006; 20 (1): 51-6. DOI: 10.1053/j.jvca.2005.03.026.

14. Chung M.K., Martin D.O., Sprecher D. et al. C-reactive protein elevation in patients with atrial arrhythmias: inflammatory mechanisms and persistence of atrial fibrillation. Circulation. 2001; 104 (24): 2886-91. DOI: 10.1161/hc4901.101760.

15. Scherer M., Sirat A., Dogan S. et al. Does totally endoscopic access for off-pump cardiac surgery influence the incidence of postoperative atrial fibrillation in coronary artery bypass grafting? A preliminary report. Cardiovasc. Engg. 2006; 6 (3): $118-21$.

16. Liakopoulos O.J., Choi Y.H., Kuhn E.W. et al. Statins for prevention of atrial fibrillation after cardiac surgery: a systematic literature review. J. Thorac. Cardiovasc. Surg. 2009; 138 (3): 678-86. DOI: 10.1016/j.jtcvs.2009.03.054.

17. Liakopoulos O.J., Kuhn E.W., Slottosch I. et al. Preoperative statin therapy for patients undergoing cardiac surgery. Cochrane
Database Syst. Rev. 2012; 4: CD008493. DOI: 10.1002/14651858.CD008493.pub2.

18. Patti G., Chello M., Candura D. et al. Randomized trial of atorvastatin for reduction of postoperative atrial fibrillation in patients undergoing cardiac surgery: results of the ARMYDA-3 (Atorvastatin for Reduction of Myocardial Dysrhythmia After cardiac surgery) study. Circulation. 2006; 114 (14): 1455-61. DOI: 10.1161/CIRCULATIONAHA.106.621763.

19. Marín F., Pascual D.A., Roldán V. et al. Statins and postoperative risk of atrial fibrillation following coronary artery bypass grafting. Am. J. Cardiol. 2006; 97 (1): 55-60. DOI: 10.1016/j.amjcard.2005.07.124.

20. Lertsburapa K., White C.M., Kluger J. et al. Preoperative statins for the prevention of atrial fibrillation after cardiothoracic surgery. J. Thorac. Cardiovasc. Surg. 2008; 135 (2): 405-11. DOI: $10.1016 /$ j.jtcvs.2007.08.049.

21. Rader F., Gajulapalli R.D., Pasala T., Einstadter D. Effect of early statin therapy on risk of atrial fibrillation after coronary artery bypass grafting with or without concomitant valve surgery. Am. J. Cardiol. 2011; 108 (2): 220-2. DOI: 10.1016/ j.amjcard.2011.03.028.

22. Liakopoulos O.J. Statins for prevention of atrial fibrillation after cardiac surgery: a systematic literature review. J. Thorac. Cardiovasc. Surg. 2009; 138 (3): 678-86.e1. DOI: 10.1016/ j.jtcvs.2009.03.054

23. Chen W.T. Effect of statins on atrial fibrillation after cardiac surgery: a duration- and dose-response meta-analysis. J. Thorac. Cardiovasc. Surg. 2010; 140 (2): 364-72. DOI: 10.1016/ j.jtcvs. 2010.02.042.

24. Lertsburapa K., White C.M., Kluger J. et al. Preoperative statins for the prevention of atrial fibrillation after cardiothoracic surgery. J. Thorac. Cardiovasc. Surg. 2008; 135 (2): 405-11. DOI: $10.1016 /$ j.jtcvs.2007.08.049.

25. Kourliouros A., De Souza A., Roberts N. et al. Dose-related effect of statins on atrial fibrillation after cardiac surgery. Ann. Thorac. Surg. 2008; 85 (5): 1515-20. DOI: 10.1016/ j.athoracsur.2008.01.040.

26. Mithani S., Akbar M.S., Johnson D.J. et al. Dose dependent effect of statins on postoperative atrial fibrillation after cardiac surgery among patients treated with beta blockers. J. Cardiothorac. Surg. 2009; 4: 61. DOI: 10.1186/ 1749-8090-4-61.

27. Jacob K.A., Nathoe H.M., Dieleman J.M. et al. Inflammation in new-onset atrial fibrillation after cardiac surgery: a systematic review. Eur. J. Clin. Invest. 2014; 44 (4): 402-28. DOI: 10.1111/eci.12237.

28. Kuhn E.W., Liakopoulos O.J., Stange S. et al. Preoperative statin therapy in cardiac surgery: a meta-analysis of 90000 patients. Eur. J. Cardiothorac. Surg. 2014; 45 (1): 17-26. DOI: 10.1093/ejcts/ezt181.

29. Mariscalco G., Lorusso R., Klersy C. et al. Observational study on the beneficial effect of preoperative statins in reducing atrial fibrillation after coronary surgery. Ann. Thorac. Surg. 2007; 84 (4): 1158-64. DOI: 10.1016/j.athoracsur.2007.05.021.

30. Zheng H., Xue S., Hu Z.L. et al. The use of statins to prevent postoperative atrial fibrillation after coronary artery bypass grafting: a meta-analysis of 12 studies. J. Cardiovasc. Pharmacol. 2014.

Поступила 29.05.2014 г. Подписана в печать 20.07.2014 г. 